\title{
125 Jahre Kümmerly+Frey und die Schweizer Geographie
}

Wenn am 8. Juni 1977 die Firma Kümmerly + Frey ihren 125 . Geburtstag feiert, gratulieren ihr mit vielen andern auch die Geographen im allgemeinen und die Geographica Helvetica im besonderen von Herzen. In Reden und Gratulationsadressen wird ihrer Leistungen gedacht werden und man wird sich an jene Männer erinnern, die aus kleinen Anfängen heraus das heutige Unternehmen geschaffen haben. Weil man heute rascher als einst lebt und sich oft frägt, wie man wohl die gegenwärtigen und zukünftigen Schwierigkeiten meistern werde, und dabei das weiter Zurückliegende vergißt, sind solche Festtage wertvoll: Man gewinnt, indem man Rückschau hält, das richtige $\mathrm{Ma} \beta$ für die Probleme der Gegenwart.

Unsere Zeitschrift hat allen Grund, aus einer solchen Rückschau Vertrauen in die Zukunft zu schöpfen. Der Zweite Weltkrieg war eben zu Ende gegangen, da trafen sich an der Türe zum Lesesaal der Zentralbibliothek Zürich Ernst Winkler und der Schreibende. Damals lag Deutschland völlig zerstört darnieder und es schien, als ob es Jahrzehnte brauche, bis aus den Trümmern wieder Leben kommen würde. «Jetzt müssen wir eine geographische Zeitschrift auf die Beine stellen, die zum mindesten den Schweizer Geographen die Möglichkeit zum Publizieren und zum wissenschaftlichen Gedankenaustausch geben kann - im Januar 1946 muß die erste Nummer erscheinen.» Noch in der gleichen Woche fanden wir einen Namen, Geographica Helvetica, und begannen mit der Arbeit. Von Anfang an schwebte uns vor, eine im wahren Sinne schweizerische Zeitschrift herauszugeben. Damals machten wir uns aber noch keine Vorstellung von den Schwierigkeiten, die sich einer solchen Absicht entgegenstellen würden. Wir dachten daran, daß durch ein Zusammenlegen der verschiedenen lokalen Zeitschriften ein tragfähiges Fundament geschaffen werden könne und übersahen, daß Lokalinteressen und schon bestehende Tauschverbindungen eine solche Entwicklung verunmöglichten. So mußte die Zeitschrift mit dem Stigma behaftet, eine zürcherische Zeitschrift zu sein, ihr Leben beginnen.

Nun hatte seit 1923 Kümmerly + Frey die kleine, regelmäßig erscheinende Zeitschrift Der Schweizer Geograph veröffentlicht, in der manch wertvoller Artikel erschienen ist. Die Herren Walter Kümmerly und Dr. Heinrich Frey sorgten sich immer besonders liebevoll um dieses im Vergleich mit anderen Erzeugnissen des Hauses unscheinbare Pflänzchen. Schon damals wurde an der Hallerstraße nicht nur das Gewinnbringende und nach außen in Erscheinung tretende umsorgt, vielmehr wurden bewußt auch weniger lukrative Leistungen für die Wissenschaft und Beiträge zum kulturellen Leben angestrebt. Bei den 1945 von Zürich ausgehenden Sondierungen nach aktiver Unterstützung der geplanten neuen Zeitschrift Geographica Helvetica fand man vorerst nur bei Kümmerly + Frey Verständnis. Man kam überein, den Schweizer Geograph und die Mitteilungen der Geographisch-Ethnographischen Gesellschaft Zürich ab 1946 zusammenzulegen und als Geographica Helvetica weiterzuführen. Die beiden Partner teilten sich seither in die manchmal stark anwachsenden Defizite und Kümmerly + Frey übernahm außerdem alle Arbeiten im Zusammenhang mit Druck, Buchhaltung und Versand, während die Gesellschaft die vom Verlag unabhängige Redaktion bestellte. Während 25 Jahren wurde die Zeitschrift von Ernst Winkler als verantwortlichem Redaktor geleitet. Damals und bis auf den heutigen Tag war von Seiten der Firma Herr Paul Etzweiler heute Vizedirektor -, seit vielen Jahren unterstützt von Herrn Isidor Bächler, für die Zeitschrift tätig, während die Herren Walter Kümmerly und Max Frey wohlwollend ihre Zustimmung zu den notwendigen Zuwendungen gaben. Dank einer konsequenten Redaktionspolitik entstand trotz der anfänglichen Schwierigkeiten eine wirklich schweizerische Zeitschrift, die sich heute der aktiven Mitarbeit und der Anerkennung aller geographischen Gesellschaften und Institutionen unseres Landes erfreut. Heute wird die Firma von den Herren Walter und Beat Frey geleitet; an der positiven Einstellung gegenüber Geographica Helvetica hat sich nichts geändert. Sollten einmal irgendwelche Schwierigkeiten auftreten, dürften allen schon die über Jahrzehnte gepflegten persönlichen und freundschaftlichen Beziehungen ermöglichen, sie zu überwinden. Nach dem Gesagten hat Geographica Helvetica allen Grund, sich heute unter die Gratulanten zum 125. Geburtstag einzureihen. Sie verbindet mit den Glückwünschen den Dank für alles bisher Geleistete und spricht die Hoffnung aus, daß die Geographen der Schweiz dank dieser Zusammenarbeit auch in $\mathrm{Zu}$ kunft ihre eigene Zeitschrift haben dürfen.

Prof. Dr. Hans Boesch, Geogr. Institut der Universität Zürich, Blümlisalpstraße 10, 8006 Zürich. 
Geographica Helvetica begann, wie gezeigt, ihr Leben im Frühsommer 1945 und erblickte das Licht der Welt anfangs 1946. Etwas früher - noch während der Kriegsjahre - faßte die Firmenleitung den Beschluß, die bisherigen Produktionszweige durch einen Buchverlag zu erweitern. Gleichzeitig verfolgte man das Ziel, durch großzügigen Ausbau des technischen Betriebes die von der Kartographie her gewohnte Präzision auch für Qualitätsdrucke des Handels und der Industrie einzusetzen. Bis dahin waren Kümmerly + Frey vor allem als kartographische Anstalt - man denke an die Schulwandkarte und die vielen Touristenkarten - nach außen in Erscheinung getreten.

Als am 1. Mai 1942 Paul Etzweiler die Aufgabe übernahm, den «Verlag» auf die Beine zu stellen, mußte er fast überall Neuland bearbeiten.

Rückschauend erscheint vor allem wichtig, daß der neue geographische Buchverlag bestrebt war, schweizerische Geographen als Autoren zu gewinnen. Anfänglich erschienen zwar nur einige wenige Bände im Laufe eines Jahres. Erfahrungen in der Produktion mußten gewonnen werden und Schritt um Schritt wurde die Marktorganisation ausgebaut. Heute sind diese Schwierigkeiten überwunden und vor allem die Erfolge der Bildbände zeigen, daß in technischer Hinsicht heute ein Stand erreicht ist, der eine Spitzenposition einnimmt. Der Markt konnte durch eine ausgebaute Verkaufsorganisation und durch Lizenzverträge so erweitert werden, daß ein Autor die Gewißheit hat, daß was er schreibt auch zur Kenntnis genommen werden wird. Wenn man daran denkt, daß jeder neue Autor ein wirtschaftliches Risiko bedeutet, darf man mit besonderer Freude festhalten, daß der Verlag von Anfang an darauf verzichtete, Lizenzausgaben von Verkaufsschlagern zu drucken oder jene
Autoren zu bevorzugen, die für die Masse zu schreiben bereit waren. Im Namen von vielen Kollegen möchte der Schreibende darum an diesem Geburtstage dankend festhalten, daß Kümmerly + Frey den Mut hatte, gar manchem die ersten schriftstellerischen Schritte zu ermöglichen, und sogar Arbeiten veröffentlichte, von denen eher zu erwarten war, daß sie Verluste bringen könnten. Mehr als einmal stellte Herr Walter Kümmerly fest: «Unsere Firma muß immer wieder wissenschaftlich wertvolle Bücher und Karten veröffentlichen, weil sie damit ihr Ansehen als Geographischer Verlag hebt - der wirtschaftliche Erfolg kann oft nicht unmittelbar und direkt gemessen werden». Heute hat der Verlag an Bedeutung so gewonnen, daß es oft schwierig ist, zur Realisierung der verlegerischen Pläne Autoren zu finden, die innerhalb der gewünschten Zeit ihr Manuskript fertigstellen können. Mit besonderer Freude sei festgehalten, daß die Tochter von Walter Kümmerly, Frau Dr. Barbara Peters-Kümmerly, als Redaktorin seit Jahren im Verlag tätig ist.

Die Firma nennt sich heute «Kümmerly + Frey, Geographischer Verlag». Daran haben wir Freude. Vielerorts haben in den vergangenen Jahren Fachkollegen das Wort «Geographie» über Bord geworfen und sich als Gesellschaftswissenschafter oder "spatial analyst» bezeichnet. Oft mag ein unangebrachtes Minderwertigkeitsgefühl dazu geführt haben - wer überzeugt ist, $\mathrm{da} ß$ er wertvolle und gute Arbeit leistet, wird auf die Frage nach seinem Beruf freilich mit Stolz: «Geograph» antworten. Wer so entscheidende Beiträge für die schweizerische Geographie wie unser Jubilar geleistet hat, darf sich zu unserer aller Freude und mit berechtigtem Stolz als «Geographischer Verlag» verstehen. 